\title{
The Mass of ${ }^{22} \mathrm{Mg}$
}

\author{
M. Mukherjee, ${ }^{1}$ A. Kellerbauer, ${ }^{2, *}$ D. Beck, ${ }^{1}$ K. Blaum, ${ }^{1,2}$ G. Bollen, ${ }^{3}$ F. Carrel, ${ }^{2}$ P. Delahaye, ${ }^{2}$ J. Dilling, ${ }^{4}$ S. George ${ }^{5}$ \\ C. Guénaut, ${ }^{6}$ F. Herfurth, ${ }^{1}$ A. Herlert, ${ }^{7}$ H.-J. Kluge, ${ }^{1}$ U. Köster, ${ }^{2}$ D. Lunney, ${ }^{6}$ S. Schwarz, ${ }^{3}$ \\ L. Schweikhard, ${ }^{7}$ and C. Yazidjian ${ }^{1}$ \\ ${ }^{1}$ GSI, Planckstraße 1, 64291 Darmstadt, Germany \\ ${ }^{2}$ Department of Physics, CERN, 1211 Genève 23, Switzerland \\ ${ }^{3}$ NSCL, Michigan State University, East Lansing, Michigan 48824-1321, USA \\ ${ }^{4}$ TRIUMF, Vancouver, British Columbia V6T 2A3, Canada \\ ${ }^{5}$ Institut für Kernphysik, Westfälische Wilhelms-Universität, 48149 Münster, Germany \\ ${ }^{6}$ CSNSM-IN2P3-CNRS, 91405 Orsay-Campus, France \\ ${ }^{7}$ Institut für Physik, Ernst-Moritz-Arndt-Universität, 17487 Greifswald, Germany
}

(Received 16 June 2004; published 8 October 2004)

\begin{abstract}
Mass measurements with a relative precision of better than $1.5 \times 10^{-8}$ were performed on ${ }^{22} \mathrm{Mg}$ and its reaction partners ${ }^{21} \mathrm{Na}$ and ${ }^{22} \mathrm{Na}$ with the ISOLTRAP Penning trap mass spectrometer at CERN, yielding the mass excesses $D\left({ }^{22} \mathrm{Mg}\right)=-399.92(27) \mathrm{keV}, \quad D\left({ }^{21} \mathrm{Na}\right)=-2184.71(21) \mathrm{keV}$, and $D\left({ }^{22} \mathrm{Na}\right)=-5181.56(16) \mathrm{keV}$. The importance of these results is twofold. First, a comparative halflife ( $F t$ value) has been obtained for the superallowed $\beta$ decay of ${ }^{22} \mathrm{Mg}$ to further test the conservedvector-current hypothesis. Second, the resonance energy for the ${ }^{21} \mathrm{Na}$ proton capture reaction has been independently determined, allowing direct comparisons of observable $\gamma$ radiation in nova explosions with the yield expected from models.
\end{abstract}

DOI: 10.1103/PhysRevLett.93.150801

The nuclear binding energy has a profound influence on a diversity of natural phenomena, ranging from the explosion of stars in the cosmos to the microscopic extreme of the interactions governing quarks. Mass differences between radioactive parent and daughter nuclides in the case of superallowed $\beta$ decays are critical for weak-interaction studies, in particular for a test of the conserved-vector-current (CVC) hypothesis and ultimately for the determination of the $V_{u d}$ element of the Cabibbo-Kobayashi-Maskawa (CKM) quark-mixing matrix. The binding energy also determines how much energy is available for a nuclear reaction, thus playing a fundamental role in the elaboration of models for stellar evolution and critical aspects of nucleosynthesis.

The short-lived radionuclide ${ }^{22} \mathrm{Mg}\left(T_{1 / 2}=3.88 \mathrm{~s}\right)$ has recently been the subject of controversy on these latter two fronts: the superallowed $\beta$ decay of ${ }^{22} \mathrm{Mg}$ to ${ }^{22} \mathrm{Na}$ [1] and the ${ }^{21} \mathrm{Na}(p, \gamma)^{22} \mathrm{Mg}$ reaction rate in classical novae [2]. In both contexts, the authors concluded that there was conflicting information on the ${ }^{22} \mathrm{Mg}$ mass. The value tabulated in the recent atomic mass evaluation (AME 2003) [3] is derived from two discrepant 30-year-old reaction $Q$ value measurements. Clearly, an independent and direct high-precision measurement of the ${ }^{22} \mathrm{Mg}$ mass with an uncertainty well below $1 \mathrm{keV}$ was required. In this Letter, we report on such a measurement, which has recently been performed with the ISOLTRAP Penning trap mass spectrometer at ISOLDE/CERN.

The CVC hypothesis holds that the (corrected) comparative half-lives $F t$ of all superallowed $\beta$ decays are equal as a consequence of the assumed independence of the vector-current part of the weak interaction from the
PACS numbers: 21.10.Dr, 23.40.Bw, 26.30.+k, 27.30.+t

strong force. The determination of $F t$ requires precision measurements of the decay energy and the partial decay half-life, as well as detailed calculations of the associated isospin-symmetry-breaking $\left(\delta_{C}\right)$ and radiative corrections $\left(\delta_{R}\right)$ [4,5]. The comparative half-lives for nine superallowed $T=1\left(0^{+} \rightarrow 0^{+}\right)$decays have been determined to a precision of $\approx 10^{-4}$ or better [5]. They agree well with each other within their uncertainties and yield a weighted mean value of $\overline{F t}=3072.2(0.9)(1.1) \mathrm{s}$, where the second uncertainty term represents a deviation in the calculation of $\delta_{C}$ by two different groups $[4,6]$ and the first term contains all other uncertainty contributions.

The vector coupling constant $G_{V}$ extracted from nuclear $\beta$ decay, together with that from muon decay [7], yields the most precise value of the $V_{u d}$ element of the CKM matrix. Using this result for $V_{u d}$, the unitarity test of the CKM matrix currently fails by more than 2 standard deviations [5], a result which is also confirmed by the $V_{u d}$ value obtained from neutron $\beta$ decay [8]. Based on the realization that the uncertainty in $V_{u d}$ is now mainly due to the calculated corrections, in particular the nuclear-structure-dependent terms, it has been pointed out [4] that these terms could be validated by measuring additional superallowed transitions covering a wider range of magnitudes of the correction terms. Of particular interest in this context are the even $Z, T_{z}=-1$ nuclei with $18 \leq A \leq 42$, and the odd $Z, T_{z}=0$ nuclei with $A \geq 62$ [4]. We have lately addressed the $A=74$ case that falls into the latter category [9]. In this Letter, we present a new result for the former $(A=22)$, for which important progress was recently made by Hardy et al. in the determination of the partial half-life [1]. 
The other domain for which the mass of ${ }^{22} \mathrm{Mg}$ is important is nuclear astrophysics. As detailed knowledge of nuclear structure is necessary but not sufficient for the constraint of stellar models, one of the most important quests in astrophysics is the definition of clear observable. Nova bursts are of particular interest due to their relative occurrence frequency and are viable candidates for observation with $\gamma$ ray telescopes, as first pointed out by Clayton and Hoyle [10]. Nucleosynthesis in ONe nova explosions proceeds by radiative proton capture on abundant ${ }^{20} \mathrm{Ne}$ seed nuclei that ultimately produces the $\beta$-decaying nuclide ${ }^{22} \mathrm{Na}$. The $\gamma$ ray in question is emitted from the first excited state of its daughter ${ }^{22} \mathrm{Ne}$ at $1275 \mathrm{keV}$. All searches so far with several different instruments have failed to detect the ${ }^{22} \mathrm{Na} 1275-\mathrm{keV} \gamma$ ray signature [11]. However, the expected frequency of $\mathrm{ONe}$ nova outbursts and the dependence of the flux on the spatial distribution have recently been revised [11], and the upper limits for the ejected ${ }^{22} \mathrm{Na}$ mass deduced from the absence of observed $\gamma$ radiation are still compatible with current nova models.

The so-called NeNa cycle for the production of ${ }^{22} \mathrm{Na}$ can follow two possible paths, shown in Fig. 1(a), depending on the ${ }^{21} \mathrm{Na}(p, \gamma)^{22} \mathrm{Mg}$ reaction rate and the temperature at the reaction site [12]. In the "cold" cycle, ${ }^{21} \mathrm{Na}$ produced in the ${ }^{20} \mathrm{Ne}(p, \gamma)^{21} \mathrm{Na}$ proton capture reaction $\beta$ decays to ${ }^{21} \mathrm{Ne}$, which forms ${ }^{22} \mathrm{Na}$ through a second $(p, \gamma)$ reaction. At higher temperatures, the proton capture on ${ }^{21} \mathrm{Na}$ is enhanced and ${ }^{22} \mathrm{Mg}$ is predominantly produced. In both cases, the $\beta^{+}$decay chain proceeds through ${ }^{22} \mathrm{Na}$ to ${ }^{22} \mathrm{Ne}$, but in the cold cycle the ${ }^{22} \mathrm{Na}$ production is delayed by the $\beta$ decay of ${ }^{21} \mathrm{Na}$, making more ${ }^{22} \mathrm{Na}$ available at a time when the nova envelope has become transparent to $\gamma$

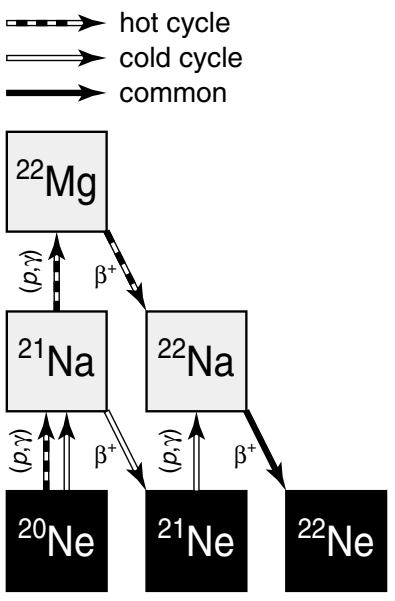

(a)

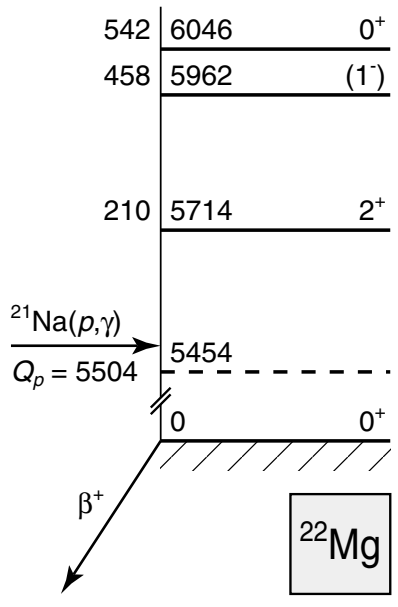

(b)
FIG. 1. (a) "Hot" and "cold" reaction paths of the $\mathrm{NeNa}$ cycle. The bottom row (black boxes) are stable nuclides; (b) level scheme of ${ }^{22} \mathrm{Mg}$, showing the levels with astrophysical relevance [31]. The level energies $E_{x}$ (center) are shown along with the center-of-mass energies $E_{x}-Q_{p}$ (left; both in $\mathrm{keV}$ ) and the spin parity (right). radiation. The quantity of ${ }^{22} \mathrm{Na}$ observable in these nova outbursts hence depends critically on the ${ }^{21} \mathrm{Na}(p, \gamma)^{22} \mathrm{Mg}$ reaction rate, which is an exponential function of the resonance energy $E_{R}[13]$.

In current ONe nova models [14,15], a precise computation of the amount of ${ }^{22} \mathrm{Na}$ ejecta is hampered by the uncertainty of the ${ }^{21} \mathrm{Na}(p, \gamma)^{22} \mathrm{Mg}$ reaction rate. Under nova conditions, it is expected to be dominated by one or several narrow resonances, in particular that into the $5714-\mathrm{keV} 2^{+}$state [12]. The resonance strength of the capture reaction can be obtained from shell structure calculations $[12,16]$. It has also recently been measured experimentally from the maximum thick-target yield by Bishop et al. [2], who found a discrepancy in the resonance energy of the $2^{+}$level which pointed to a probable deviation of the ${ }^{22} \mathrm{Mg}$ mass from the AME value.

To resolve this double discrepancy concerning the mass of ${ }^{22} \mathrm{Mg}$, we have performed Penning trap mass measurements with ISOLTRAP [17] at the online isotope separator facility ISOLDE [18] at CERN. The ISOLTRAP apparatus consists of a gas-filled linear Paul trap for beam preparation [19], a cylindrical Penning trap for the cooling and isobaric cleaning of the ion bunch, and a hyperbolical Penning trap for the precision mass measurements [17]. A mass measurement is carried out via the determination of the cyclotron frequency $\nu_{c}=$ $q B /(2 \pi m)$ of the ion in the precision Penning trap using the time-of-flight resonance technique [20,21], where $q$ and $m$ are the charge and the mass of the ion and $B$ is the magnetic-field magnitude. The ion's mass is obtained from the comparison of its cyclotron frequency $\nu_{c}$ with that of a well-known "reference mass." The resolving power achieved in the precision Penning trap is given by the product of the excitation time $t_{\mathrm{exc}}$ and the cyclotron frequency $\nu_{c}$. Calibration measurements using a carbon cluster ion source have shown that the overall accuracy of ISOLTRAP for the frequency ratio of any two nuclides is $8 \times 10^{-9}$ [22].

For the measurements reported here, magnesium and sodium atoms were produced by bombarding a $19-\mathrm{g} / \mathrm{cm}^{2}$ Ti foil target (rolls of $30-\mu \mathrm{m}$ foil) with $1.4-\mathrm{GeV}$ protons from the CERN Proton Synchrotron Booster. The $\mathrm{Mg}$ isotopes were selectively laser ionized with the ISOLDE resonance ionization laser ion source [23] and $\mathrm{Na}$ was thermally ionized in the $2100{ }^{\circ} \mathrm{C}$ hot $\mathrm{W}$ ionizer. ${ }^{21} \mathrm{Na}$ was measured in a separate run, produced from a standard ISOLDE $\mathrm{UC}_{x} /$ graphite target with a W surface ionizer. The ions were accelerated to $60 \mathrm{keV}$ and mass separated in the high-resolution mass separator (HRS). The yield of ${ }^{22} \mathrm{Mg}$ was $3.1 \times 10^{5} / \mu \mathrm{C}$, that of ${ }^{22} \mathrm{Na}$ was about $10^{9} / \mu \mathrm{C}$. For ${ }^{22} \mathrm{Mg}$ the HRS was operated at a mass resolving power of $m / \delta m \approx 4000$ in order to suppress the more abundant ${ }^{22} \mathrm{Na}$, improving the ratio of ${ }^{22} \mathrm{Mg}$ to ${ }^{22} \mathrm{Na}$ to about $1 / 200$. The suppression of the remaining contaminants was achieved in ISOLTRAP's cooling Penning trap. Any residual contamination still present in the precision Penning trap would lead to a cyclotron frequency 
shift which increases with an increased number of ions stored simultaneously. Such an effect was excluded in the course of the standard analysis procedure [22] in which no such dependence was found for any of the studied nuclides. In the case of ${ }^{22} \mathrm{Mg}$ with a potential ${ }^{22} \mathrm{Na}$ contamination, the number of ions in the trap was less than one on average, effectively eliminating any such effect. Over the measurement period of about three days, eight resonances of ${ }^{22} \mathrm{Mg}$ were recorded, of which a typical example is shown in Fig. 2(a).

The results of the data taking period are summarized in Table I. The table gives the frequency ratios of the reference nuclides to the ions of interest as well as the mass excesses of the studied nuclides. The uncertainties of the averaged frequency ratios include contributions due to a magnetic-field drift between the two reference measurements and the mass difference between the ion of interest and the reference ion, as well as the residual systematic uncertainty. The mass excesses were obtained from the frequency ratios by means of a least-squares adjustment [24] very similar to that employed in the
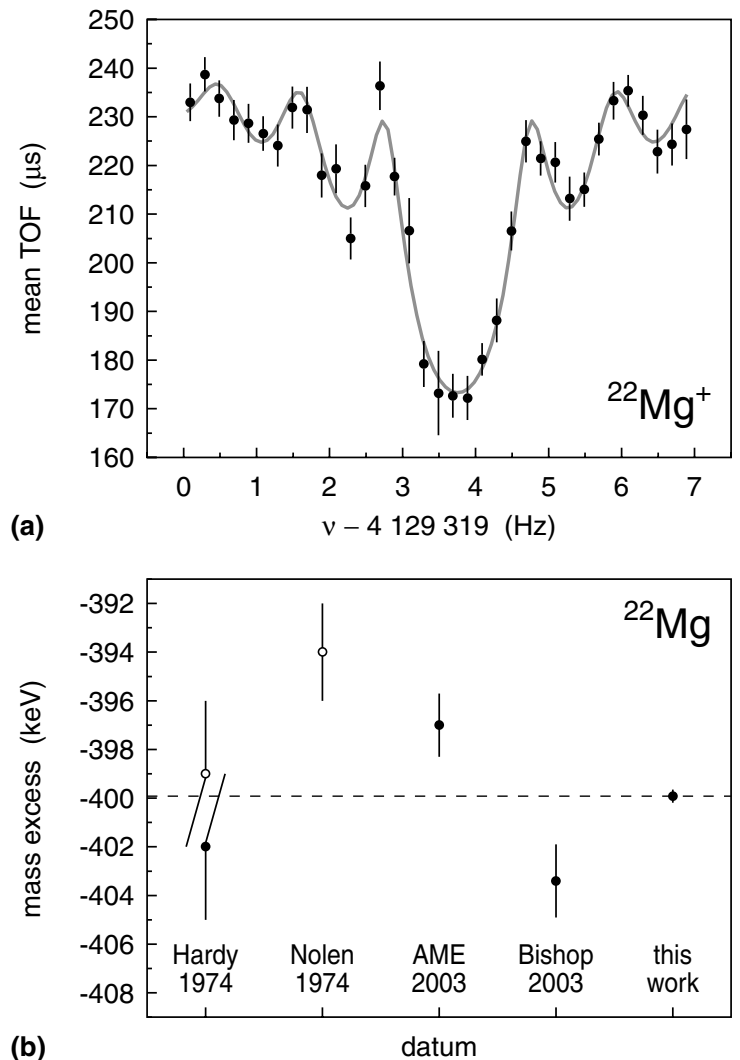

FIG. 2. (a) Typical time-of-flight (TOF) cyclotron resonance of ${ }^{22} \mathrm{Mg}^{+}$. The solid line is a fit of the theoretically expected line shape [21] to the data; (b) comparison of the mass excess value from the measurement reported here with previous results and the AME 2003 [3]. For Ref. [29], both the original (open circle) and the recalibrated (solid circle) [1] values are shown. The dashed line is the central value of our result.
AME [25]. This technique allowed us to simultaneously take into account the 16 frequency and mass relations recorded in this data taking period (only 6 of the 10 frequency relations are shown in Table I). We slightly increased the residual systematic uncertainty added to the frequency relations from $8 \times 10^{-9}$ as found in Ref. [22] to $1.3 \times 10^{-8}$ in order to obtain a reduced $\chi^{2}$ of the adjustment of $\chi^{2} / 9=1$. This assures the overall consistency of our measurements, both internally and with the high-precision data to which they are connected. The mass excess for ${ }^{21} \mathrm{Na}$ obtained in this experiment is in excellent agreement with the value from the AME 2003 [3]. Our result for the mass of ${ }^{22} \mathrm{Na}$ agrees well with a value deduced from ${ }^{21} \mathrm{Ne}(p, \gamma)^{22} \mathrm{Na}$ resonances [26]. It disagrees by $2.3 \sigma$ with the weighted mean of two $\beta$ end point measurements using the ${ }^{22} \mathrm{Na}\left(\beta^{+}\right)^{22} \mathrm{Ne}$ decay [27,28]. A comparison of our result for the ${ }^{22} \mathrm{Mg}$ mass excess with previous data is shown in Fig. 2(b). The mass excess of ${ }^{22} \mathrm{Mg}$ of AME 2003 is derived from two $(p, t)$ reaction $Q$ values [29,30] for which the reaction energies of the corresponding calibration reactions have changed in the meantime [open circles in Fig. 2(b)]. While a recalibration of the value of Ref. [30] appears not to be feasible, the other [29] was updated by Hardy et al. [1] (solid circle), resulting in a lower mass excess. The result from Ref. [2] also indicated that ${ }^{22} \mathrm{Mg}$ was probably more bound, a conclusion that is confirmed by our measurement. In addition to the mass excesses of the studied nuclides, we have also extracted the mass excess difference $D\left({ }^{22} \mathrm{Mg}\right)-D\left({ }^{22} \mathrm{Na}\right)=4781.64(28) \mathrm{keV}$ directly from the least-squares adjustment.

From this mass excess difference and the level energy $E_{x}=657.00(14) \mathrm{keV}$ for the $0^{+}$state in ${ }^{22} \mathrm{Na}$ [31], we obtain a decay energy for the superallowed decay of ${ }^{22} \mathrm{Mg}$ of $Q=4124.64(31) \mathrm{keV}$, which corresponds to a statistical rate function $f=418.48(19)$ [32]. Using the halflife $T_{1 / 2}=3.8755(12) \mathrm{s}$, the branching ratio into the superallowed channel $R=53.15(12) \%$ [1], the corrections $\delta_{R}=1.20(3) \%$ and $\delta_{C}=0.265(15) \%$ [4], as well as an electron capture fraction of $P_{\mathrm{EC}}=0.07 \%$ [32], this

TABLE I. Frequency ratios $\nu_{c}^{\mathrm{ref}} / \nu_{c}$ (with respect to the indicated reference nuclide), mass excesses $D$, and mass excess literature values $D_{\text {lit }}$ [3] for the nuclides ${ }^{21} \mathrm{Na},{ }^{22} \mathrm{Na}$, and ${ }^{22} \mathrm{Mg}$.

\begin{tabular}{lccc}
\hline \hline Nuclide-Reference & $\nu_{c}^{\text {ref }} / \nu_{c}$ & $\begin{array}{c}D^{\mathrm{a}} \\
(\mathrm{keV})\end{array}$ & $\begin{array}{c}D_{\text {lit }} \\
(\mathrm{keV})\end{array}$ \\
\hline${ }^{21} \mathrm{Na}^{23} \mathrm{Na}$ & $0.9133457067(140)$ & & \\
${ }^{21} \mathrm{Na}-{ }^{39} \mathrm{~K}$ & $0.53889641265(737)$ & $-2184.71(21)$ & $-2184.20(70)$ \\
${ }^{22} \mathrm{Na}-{ }^{23} \mathrm{Na}$ & $0.9567044060(127)$ & & \\
${ }^{22} \mathrm{Na}-{ }^{24} \mathrm{Mg}$ & $0.9170045076(130)$ & & \\
${ }^{22} \mathrm{Na}^{39} \mathrm{~K}$ & $0.56447912311(769)$ & $-5181.56(16)$ & $-5182.40(40)$ \\
${ }^{22} \mathrm{Mg}^{22} \mathrm{Na}$ & $1.0002333826(156)$ & & \\
${ }^{22} \mathrm{Mg}-{ }^{39} \mathrm{~K}$ & $0.56461086300(897)$ & $-399.92(27)$ & $-397.0(1.3)$ \\
\hline
\end{tabular}

${ }^{\mathrm{a}}$ Using $\quad D\left({ }^{23} \mathrm{Na}\right)=-9529.8536(27) \mathrm{keV}, \quad D\left({ }^{24} \mathrm{Mg}\right)=$ $-13933.567(13) \mathrm{keV}, \quad D\left({ }^{39} \mathrm{~K}\right)=-33807.01(19) \mathrm{keV}, m_{e}=$ 510.998 903(4) $\mathrm{keV}$, and $1 \mathrm{u}=931494.009(7) \mathrm{keV}$ [3].

150801-3 


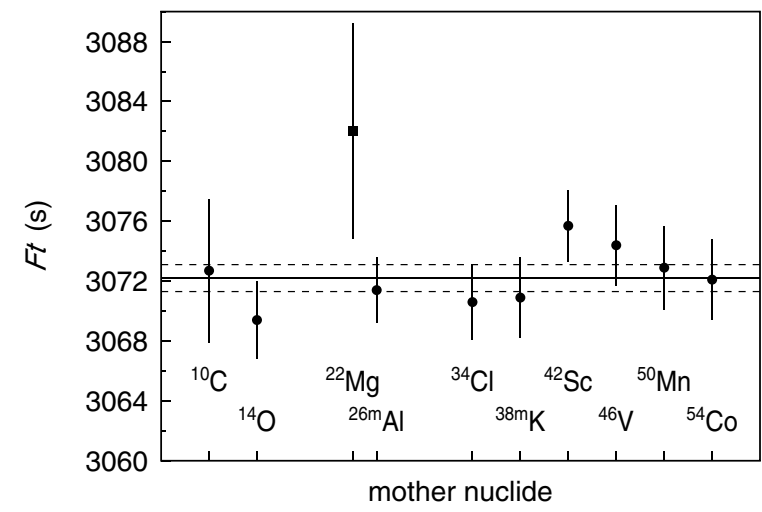

FIG. 3. Comparative half-life for the decay ${ }^{22} \mathrm{Mg}\left(\beta^{+}\right)^{22} \mathrm{Na}$ (square) compared with the high-precision data for the other nine nuclides ${ }^{10} \mathrm{C}-{ }^{54} \mathrm{Co}$ (circles) [5]. The dashed lines indicate the confidence interval of the weighted mean $\overline{F t}=3072.2(9) \mathrm{s}$ of the other data.

yields a comparative half-life of $F t=3082.0(7.2) \mathrm{s}$. Figure 3 shows this result alongside the existing highprecision data [5] and illustrates the agreement to within $1.3 \sigma$ with their weighted mean $\overline{F t}=3072.2(9) \mathrm{s}$. The uncertainty is still about a factor 2.5 larger than those of the other nuclides, but this is now almost entirely due to the uncertainty of the branching ratio into the superallowed channel ( $94 \%$ contribution to the variance of $F t$ ). In fact, a precise measurement of $R$ could improve the precision of $F t$ to better than $2 \mathrm{~s}$. Under the assumption of CVC, we predict a branching ratio of $R=53.319(38) \%$.

Our mass measurements on ${ }^{21} \mathrm{Na}$ and ${ }^{22} \mathrm{Mg}$ yield a ${ }^{21} \mathrm{Na}(p, \gamma){ }^{22} \mathrm{Mg}$ resonance energy into the $5713.9-\mathrm{keV}$ $2^{+}$state of $E_{R}=209.7(1.2) \mathrm{keV}$, somewhat higher than the value of 205.7(5) keV from the yield curve measurement [2]. A further reduction in the uncertainty of the resonance energy is currently hampered by the inadequate knowledge of the level energy of the aforementioned $2^{+}$state in ${ }^{22} \mathrm{Mg}$. Our measurement indicates a slightly lower ${ }^{21} \mathrm{Na}(p, \gamma){ }^{22} \mathrm{Mg}$ reaction rate at all temperatures than found in Ref. [2], but well within the uncertainties reported there. Our result therefore confirms their conclusion that the predicted final ${ }^{22} \mathrm{Na}$ content in ONe nova ejecta, and hence the $\gamma$ ray flux, does not contradict the upper limits from observational searches. In addition to the independent determination of the resonance energy, our result should allow more detailed calculations of the resonance strength of the ${ }^{21} \mathrm{Na}(p, \gamma)^{22} \mathrm{Mg}$ capture reaction as a complementary input for nova models.

The authors thank the ISOLDE technical group for their assistance. This work was supported by the German Federal Ministry for Education and Research (BMBF) under Contract No. 06GF151, by the Helmholtz Association of National Research Centres (HGF) under Contract No. VH-NG-037, by the European Commission under Contracts No. HPRI-CT-
2001-50034 (NIPNET) and No. HPRI-CT-2001-50033 (TARGISOL), and by the French IN2P3.

Note added in proof.-The masses of ${ }^{22} \mathrm{Mg}$ and ${ }^{22} \mathrm{Na}$ were recently also measured with the CPT experiment [33]. Their results agree with ours within their roughly threefold larger uncertainties.

*To whom correspondence should be addressed. Electronic address: a.kellerbauer@cern.ch

[1] J. C. Hardy et al., Phys. Rev. Lett. 91, 082501 (2003).

[2] S. Bishop et al., Phys. Rev. Lett. 90, 162501 (2003).

[3] A. H. Wapstra, G. Audi, and C. Thibault, Nucl. Phys. A729, 129 (2003).

[4] I. S. Towner and J. C. Hardy, Phys. Rev. C 66, 035501 (2002).

[5] I. S. Towner and J. C. Hardy, J. Phys. G 29, 197 (2003).

[6] W. E. Ormand and B. A. Brown, Phys. Rev. C 52, 2455 (1995).

[7] R. E. Shrock and L.-L. Wang, Phys. Rev. Lett. 41, 1692 (1978).

[8] H. Abele et al., Phys. Rev. Lett. 88, 211801 (2002).

[9] A. Kellerbauer et al., Phys. Rev. Lett. 93, 072502 (2004)

[10] D. D. Clayton and F. Hoyle, Astrophys. J. 187, L101 (1974).

[11] P. Jean et al., in Proceedings of the Fourth INTEGRAL Workshop (ESA, Noordwijk, 2001), Vol. SP-459, p. 73.

[12] M. Wiescher et al., Astron. Astrophys. 160, 56 (1986).

[13] W. A. Fowler et al., Annu. Rev. Astron. Astrophys. 5, 525 (1967).

[14] J. José, A. Coc, and M. Hernanz, Astrophys. J. 520, 347 (1999).

[15] N. A. Smirnova and A. Coc, Phys. Rev. C 62, 045803 (2000).

[16] M. Wiescher and K. Langanke, Z. Phys. 325, 309 (1986).

[17] G. Bollen et al., Nucl. Instrum. Methods Phys. Res., Sect. A 368, 675 (1996).

[18] E. Kugler, Hyperfine Interact. 129, 23 (2000).

[19] F. Herfurth et al., Nucl. Instrum. Methods Phys. Res., Sect. A 469, 254 (2001).

[20] G. Gräff, H. Kalinowsky, and J. Traut, Z. Phys. A 297, 35 (1980).

[21] M. König et al., Int. J. Mass Spectrom. Ion Processes 142, 95 (1995).

[22] A. Kellerbauer et al., Eur. Phys. J. D 22, 53 (2003).

[23] V. N. Fedoseyev et al., Hyperfine Interact. 127, 409 (2000).

[24] M. Mukherjee et al. (to be published).

[25] G. Audi, W. G. Davies, and G. E. Lee-Whiting, Nucl. Instrum. Methods Phys. Res., Sect. A 249, 443 (1986).

[26] A. Anttila, M. Bister, and E. Arminen, Z. Phys. A 234, 455 (1970).

[27] E. Beck and H. Daniel, Z. Phys. A 216, 229 (1968).

[28] H. J. Gils et al., Nucl. Instrum. Methods 105, 179 (1972).

[29] J. C. Hardy et al., Phys. Rev. C 9, 252 (1974).

[30] J. A. Nolen, Jr. et al., Nucl. Instrum. Methods 115, 189 (1974).

[31] P. M. Endt, Nucl. Phys. A521, 1 (1990).

[32] I. S. Towner and J. C. Hardy (private communication).

[33] G. Savard et al., Phys. Rev. C (to be published). 